\title{
PELP1/MNAR suppression inhibits proliferation and metastasis of endometrial carcinoma cells
}

\author{
JING WAN and XIAOMAO LI \\ Department of Gynecology and Obstetrics, The Third Affiliated Hospital, \\ Sun Yat-sen University, Guangzhou, Guangdong 510630, P.R. China
}

Received May 16, 2012; Accepted July 17, 2012

DOI: 10.3892/or.2012.2038

\begin{abstract}
Proline-, glutamic acid- and leucine-rich protein-1/ modulator of non-genomic activity of estrogen receptor (ER) (PELP1/MNAR) is a novel nuclear receptor (NR) co-activator that plays an essential role in the actions of ER. Emerging findings suggest that PELP1/MNAR is a novel proto-oncogene, whose expression is deregulated in several hormone-responsive cancers, including endometrial cancer. In this study, we demonstrate that PELP1/MNAR is widely expressed in endometrial carcinoma cell lines. To investigate its possible role in endometrial carcinoma progression, we adopted an RNA interference technology to downregulate PELP1/MNAR expression in Ishikawa endometrial carcinoma cells. PELP1/MNAR downregulation substantially reduced cell proliferation, and the cells in which PELP1/MNAR expression was knocked down also exhibited a decreased migration and invasion ability, as shown by Boyden chamber and invasion assays. The results showed that the expression of MMP-2 and MMP-9 was also decreased. These results suggest that PELP1/MNAR plays a role in endometrial cancer progression and metastasis, and that PELP1/ MNAR may be a potential therapeutic target for endometrial cancer.
\end{abstract}

\section{Introduction}

Endometrial carcinoma is one of the most common malignancies in women, and its incidence has recently increased in both developed and developing countries (1). Approximately 70-80\% of human endometrial cancers begin as hormone-dependent, and long-term estrogen stimulation without progesterone counteraction plays an important role in tumorigenesis $(2,3)$. However, the molecular basis of endometrial carcinoma remains poorly understood.

Correspondence to: Dr Xiaomao Li, Department of Gynecology and Obstetrics, The Third Affiliated Hospital, Sun Yat-sen University, 600 Tian He Road, Guangzhou, Guangdong 510630, P.R. China E-mail: tigerlee777@126.com

Key words: endometrial carcinoma, proline-, glutamic acid- and leucine-rich protein-1/modulator of non-genomic activity of estrogen receptor, co-activator, metastasis
The biological effects of estrogen are mediated by the estrogen receptor (ER), which has 2 subtypes, ER $\alpha$ and $\operatorname{ER} \beta(4,5)$. Emerging evidence suggests that ER is a key transcriptional regulator in hormone-associated cancer biology, including endometrial cancer. The mechanisms of ER are complex and involve genomic as well as non-genomic signaling events $(6,7)$. Upon binding to E2, ligand-activated ERs bind to ER-responsive elements and modulate the respective gene expression (genomic signaling), and ERs also participate in transcription-independent functions (non-genomic action) through the activation of cytosolic signaling pathways, including the Src, MAPK and protein kinase B (AKT) pathways (7-9). ER function is modulated by co-regulators $(10,11)$. Advances in research during the past decade have identified several novel proteins as being ER co-regulators, with each co-regulator playing an important and a non-overlapping function (10-18).

Proline-, glutamic acid- and leucine-rich protein-1; also known as modulator of non-genomic activity of ER (PELP1/ MNAR), is a novel ER co-activator that plays an essential role in the mechanisms of ER (19). Compared with other ER co-regulators, PELP1/MNAR is unique, as it modulates the function of $E R \alpha$ and $E R \beta$ and is involved in genomic as well as non-genomic signaling events (20-22). PELP1 is also a general co-regulator for a number of nuclear receptors (NRs), including ER, ER-related receptor (ERR), progesterone receptor (PR), glucocorticoid receptor (GR), androgen receptor (AR) and transcription factors, such as E2F, four and a half LIM domain protein 2 (FHL2) and signal transducer and activator of transcription 3 (STAT3) $(23,24)$. Emerging findings suggest that PELP1/MNAR is a novel proto-oncogene. Its expression is deregulated in several hormone-responsive cancers, including breast, ovary, endometrial and prostate cancer (24-28). The overexpression of PELP1/MNAR in fibroblasts and epithelial model cells results in cellular transformation and breast cancer cells stably overexpressing PELP1/MNAR have shown rapid tumor growth in xenograft studies (25). Endogenous PELP1/ MNAR is also required for optimal ligand-mediated transcription and proliferation responses in endometrial cancer cells (27). However, the molecular mechanism(s) responsible for its oncogenic function in endometrial carcinoma remains unclear.

In this study, we examined PELP1/MNAR expression in various endometrial cancer cell lines, and used a lentiviral vector-based RNA interference expression system targeting 
PELP1/MNAR to obtain a stable transcript knockdown and a high efficiency of RNAi delivery. We aimed to determine the effect of the downregulation of PELP1/MNAR on the proliferation, migration and invasive potential of endometrial cancer cells with or without estrogen stimulation in an attempt to provide a new strategy for hormonal carcinogenesis prevention and new therapy opportunities for the treatment of endometrial carcinoma.

\section{Materials and methods}

Reagents. PELP1/MNAR antibodies for western blot analysis were purchased from Abicam (Beverly, MA, USA), PELP1/MNAR antibodies for immunocytochemistry were purchased from Novus Biologicals (Littleton, CO, USA), horseradish, peroxidase (HRP)-conjugated goat anti-rabbit secondary antibodies were purchased from Santa Cruz Biotechnology Inc., (Santa Cruz, CA, USA) and GAPDH was purchased from Biosynthesis Technology (Beijing, China). ER $\alpha$ and ER $\beta$ antibodies were purchased from Dako (Glostrup, Denmark). 17-Estradiol (purity $\geq 98 \%$; cat. no. E8875) was purchased from Sigma Chemicals (St. Louis, MO, USA).

HEC-1A and HEC-1B human endometrial carcinoma cells were obtained from the American Type Culture Collection (Manassas, VA, USA). AN3CA and RL-952 cells were provided by the Shanghai Cell Collection, Chinese Academy of Sciences (Shanghai, China). Ishikawa human endometrial carcinoma cells were kindly provided by Professor Zehua Wang (Department of Obstetrics and Gynecology, Union Hospital, Tongji Medical College, Huazhong University of Science and Technology, Wuhan, China). The cells were grown in Dulbecco's modified Eagle's medium with essential amino acid solution (Gibco, Carlsbad, CA, USA) without phenol red and supplemented with $10 \%$ fetal calf serum (HyClone, South Logan, UT, USA).

Western blot analysis. To detect the expression of PELP1/ MNAR protein in different endometrial carcinoma cell lines, we performed western blot analysis, and used cervical cancer HeLa cell as the control. Ishikawa, HEC-1A, HEC-1B, AN3CA, RL-952 and HeLa cells were lysed using the KeyGen Total Protein Extraction kit (KeyGen, Nanjing, China) according to the manufacturer's instructions. The lysates were centrifuged at $4{ }^{\circ} \mathrm{C} 14,000 \mathrm{rpm}$ for $20 \mathrm{~min}$. The supernatants were collected and the concentrations of the supernatants were measured with a BCA protein assay kit (Pierce, Rockford, IL, USA). Protein (25-30 $\mu \mathrm{g}$ ) was applied per lane and separated by $8-12 \%$ SDS polyacrylamide gel electrophoresis, then transferred onto polyvinylidene difluoride membranes (Millipore, Billerica, MA, USA). The membranes were incubated with blocking buffer for $60 \mathrm{~min}$ at room temperature. The blocking buffer consisted of $5 \%$ non-fat dry milk dissolved in Tris-buffered saline containing $0.1 \%$ Tween-20 (TBS-T). After washing the membranes with TBS-T, they were immunoblotted overnight at $4^{\circ} \mathrm{C}$ and were incubated with rabbit anti-human PELP1/ MNAR (1:300), at $4^{\circ} \mathrm{C}$ overnight. The membranes were washed with TBS-T 3 times, and subjected to HRP-conjugated secondary antibody for $60 \mathrm{~min}$ at room temperature. Proteinantibody complexes were visualized with an ECL western blot detection system (Pierce). Western blot films were digitized, net band intensities were quantified using the Quantity One Image System and GAPDH expression levels were used to further normalize the loading amount. Each experiment was repeated 3 times to assess the consistency of the results.

Immunocytochemistry. For immunocytochemical staining, the Ishikawa, HEC-1A and AN3CA cells were plated on coverslips and cultured for $48 \mathrm{~h}$. The coverslips were fixed in $4 \%$ paraformaldehyde for $30 \mathrm{~min}$ at room temperature, washed in PBS, and permeabilized for 10 min with $0.25 \%$ Triton $\mathrm{X}-100$ in PBS. The cell coverslips were then incubated with $0.3 \%$ $\mathrm{H}_{2} \mathrm{O}_{2}$ in PBS for $15 \mathrm{~min}$. After washing in PBS, the samples were then incubated with rabbit polyclonal anti-human PELP1 antibody (1:500), mouse anti-human ER $\alpha$ antibody (1:60), or rabbit anti $\operatorname{ER} \beta$ (1:60) for $1 \mathrm{~h}$ in a humid chamber. After washing with PBS, the sections and coverslips were overlaid with Dako REAL EnVision HRP rabbit/mouse antibody at $37^{\circ} \mathrm{C}$ for $30 \mathrm{~min}$. The sections and coverslips were then counterstained with hematoxylin. Positive reactions were detected by incubating the slides with 3,3'-diaminobenzidine tetrahydrochloride for $1 \mathrm{~min}$. The immunocytochemical results were evaluated by a pathologist.

Construction and transfection of short hairpin (shRNA) lentivirus vectors. DNA template oligonucleotides corresponding to the PELP1/MNAR gene (GenBank NM_014389) were designed (28) and synthesized. The targeted sequences were: PELP1/MNAR-shRNA, 5'-GGACCAAGGTGTATGCG ATAT-3'; the sequence was inserted into the AgeI and EcoRI enzyme sites of the pGCSIL-GFP vector. The negative control (NC) sequences were 5'-TTCTCCGAACGTGTCACGT-3'. The lentiviral particles were produced using a Lentiviral Vector Expression System (Auragene, Changsha, China). Briefly, the vectors with helper plasmids were transfected into 293T cells using the calcium phosphate transfection method. The supernatant containing lentiviral particles was collected and concentrated by ultracentrifugation. The condensed lentiviral particle solution was tittered on 293T cells with the final titer $\sim 5 \times 10^{8} \mathrm{TU} / \mathrm{ml}$.

For transfection, $2 \times 10^{5}$ cells/well were seeded in a 6-well plate for $24 \mathrm{~h}$. Cells were then transfected with lentivirusmediated shRNA targeting PELP1/MNAR or negative control lentivirus vector. The multiplicity of infection (MOI) was 50. Cells transfected with lentivirus-mediated shRNA targeting PELP1/MNAR, negative control and with no transfection were named Ishikawa-KD, Ishikawa-NC and Ishikawa-CON cells, respectively. The transfection efficiency was evaluated by fluorescence microscopy and flow cytometry.

Real-time PCR. Total RNA was isolated from the cells using TRIzol (Invitrogen) according to the manufacturer's instructions. First-strand cDNA was synthesized using a PrimeScript ${ }^{\mathrm{TM}}$ RT reagent kit (Perfect Real-Time; Takara, Beijing, China). The reverse transcription (RT) reaction mixture for first-strand cDNA synthesis included 5X PrimeScript ${ }^{\mathrm{TM}}$ Buffer $2 \mu \mathrm{l}$, PrimeScript $^{\mathrm{TM}}$ RT Enzyme mix I $0.5 \mu \mathrm{l}$, Oligo(dT) primer 25 pmol, random 6 mers 50 pmol, total RNA 500 ng and RNase free $\mathrm{dH}_{2} \mathrm{O}$ in a final volume of $10 \mu \mathrm{l}$. The samples were placed on ice before reverse transcription. Then tubes were incubated for $15 \mathrm{~min}$ at $37^{\circ} \mathrm{C}, 5 \mathrm{sec}$ at $85^{\circ} \mathrm{C}$ and then chilled to $4^{\circ} \mathrm{C}$. 
Table I. Primer sequences for real-time PCR.

\begin{tabular}{lll}
\hline mRNA & \multicolumn{1}{c}{ Primer sequence } \\
\hline$\beta$-actin & Sense: & 5'-CAGCCATGTACGTTGCTATCCAGG-3' \\
& Antisense: & 5'-CAGCCATGTACGTTGCTATCCAGG-3' \\
PELP1 & Sense: & 5'-CTCAGTAATGCACGTCTCAGTTCCA-3' \\
& Antisense: & 5'-GAATGCTCCGAAGCCAAGACA-3' \\
MMP-9 & Sense: & 5'-ACGCACGACGTCTTCCAGTA-3' \\
& Antisense: & 5-CCACCTGGTTCAACTCACTCC-3' \\
MMP-2 & Sense: & 5'-CTTCCAAGTCTGGAGCGATGTG-3' \\
& Antisense: & 5'-ATGAGCCAGGAGTCCGTCCTTA-3' \\
\hline
\end{tabular}

PCR was carried out in a $20 \mu \mathrm{l}$ final volume containing the following: $2 \mu \mathrm{l}$ cDNA diluted in RNase-free water; $10 \mu \mathrm{l} \mathrm{SYBR}$ premix Ex Taq; the antisense and sense primers $0.5 \mu \mathrm{l}$ separately; ROX Reference dye II (50X) $0.4 \mu \mathrm{l}$ and $\mathrm{dH}_{2} \mathrm{O}$ in order to reach a $20 \mu \mathrm{l}$ final volume. After an initial denaturation step at $95^{\circ} \mathrm{C}$ for $30 \mathrm{sec}$, temperature cycling was initiated. Each cycle consisted of denaturation at $95^{\circ} \mathrm{C}$ for $3 \mathrm{sec}$ and elongation at $60^{\circ} \mathrm{C}$ for $30 \mathrm{sec}$. A total of 40 cycles was performed. The fluorescent signal was acquired at the end of the elongation step. Real-time PCR was performed in a ABI 7500 Fast Real-Time PCR System (Applied Biosystems, Foster City, CA, USA) with specific real-time PCR primers for each gene (Table I). Results were normalized to the actin transcript levels and the difference in fold-expression was calculated using the $\Delta \Delta \mathrm{CT}$ method. The results were representative of 3 independent experiments. Data are shown as the means \pm SD. The primers for PELP1/MNAR, $\beta$-actin, and MMPs are shown in Table I.

Cell proliferation assays. The cell viability and proliferation were measured by a 3-(4,5-dimethylthiazol-2-yl)-2,5-diphenyltetrazolium bromide (MTT) assay (Sigma, USA). Seven days after the transfection the 3 types of Ishikawa cells were seeded into 596 -well culture plates at $3.0 \times 10^{3} /$ well, with each group consisting of 5 parallel wells. After $24 \mathrm{~h}$, the cells were treated with or without E2 $\left(10^{-9} \mathrm{~mol} / \mathrm{l}\right)$. For the following 5 days, $20 \mu \mathrm{l}$ MTT $(5 \mathrm{mg} / \mathrm{ml})$ were added to each well and the cells were incubated at $37^{\circ} \mathrm{C}$ for an additional $4 \mathrm{~h}$. The reaction was terminated by lysing the cells with $150 \mu \mathrm{l}$ DMSO for $10 \mathrm{~min}$. Absorbance was measured at $490 \mathrm{~nm}$ using a microplate reader (Bio-Tek, Winooski, VT, USA). Cell growth curves were drawn by the mean optical density (OD) values.

In vitro anchorage-dependent colony-forming assay. Five days after the transfection, the 3 types of Ishikawa cells were plated in $10-\mathrm{cm}$ culture dishes at a density of $3 \times 10^{3} / \mathrm{dish}$, respectively. After $24 \mathrm{~h}$, the cells were treated with or without E2 $\left(10^{-9} \mathrm{~mol} / \mathrm{l}\right)$. The cells were fed every 2 days, and the foci of the transformed cells were counted after 14 days. The cells were stained with crystal violet and visible colonies were manually counted. The data are representative of 3 independent experiments performed in triplicate.

Cell migration and invasion. Cell migration and invasion assays were all performed in a 24-well, Transwell chamber with a $8.0-\mu \mathrm{m}$ pore membrane (Corning, Corning, NY, USA). For the cell migration assay, 3 types of Ishikawa cells $\left(5 \times 10^{4}\right)$ were seeded in the upper chamber with serum-free medium, and $0.75 \mathrm{ml}$ of complete growth medium containing $10 \%$ fetal bovine serum with or without E2 $\left(10^{-9} \mathrm{~mol} / \mathrm{l}\right)$ was added to each well in the lower chamber (21). For the invasion assays, the upper chambers were coated with 20\% Matrigel (100 $\mu \mathrm{l} /$ well) (BD Biosciences, San Diego, CA, USA). The cells were then seeded in the chamber at $1 \times 10^{5} /$ well and treated as described above. After $24 \mathrm{~h}$ of incubation at $37^{\circ} \mathrm{C}$ in a $5 \%$ $\mathrm{CO}_{2}$ atmosphere, cells on the upper side of the chamber were removed and cells invaded to the lower side were fixed, stained and counted in 10 random fields at magnification, $\mathrm{x} 100$.

Statistics analysis. All assays were conducted 3 times and found to be reproducible. Data are expressed as the means \pm SD. Comparisons among the groups were performed with the one-way analysis of variance (ANOVA) test with Bonferroni's correction for multiple comparisons. All statistical analyses were performed by using SPSS 13.0. A P-value $<0.05$ was considered to indicate a statistically significant difference.

\section{Results}

Expression profile of PELPI/MNAR in endometrial cancer cell lines. To determine whether PELP1/MNAR plays a role in endometrial cancer, we first measured the PELP1/MNAR expression in 5 commonly used endometrial cancer cell lines (Ishikawa, HEC-1A, AN3CA, HEC-1B and RL-952) and cervical cancer HeLa cells using western blot analysis. The results revealed that PELP1/MNAR was widely expressed in all the cell lines (Fig. 1). We then used immunocytochemistry to detect PELP1/MNAR as well as ER $\alpha$ and ER $\beta$ expression and location in the Ishikawa, HEC-1A and AN3CA endometrial cancer cells, and found that the expression of $\mathrm{ER} \alpha$ and ER $\beta$ varied among the cells. Ishikawa cells were ER $\alpha$-positive, HEC-1A cells were ER $\beta$-positive, whereas ER $\alpha$ and ER $\beta$ expression was low/undetectable in AN3CA cells (Fig. 2). However, all the cell lines were stained with PELP1/ MNAR and the protein was mostly located in the nucleus, which implies a potentially important role for PELP1/MNAR in endometrial cancer. Therefore, we further investigated the effects of PELP1/MNAR suppression on the Ishikawa endometrial cancer cell line and the signaling pathway involved. 


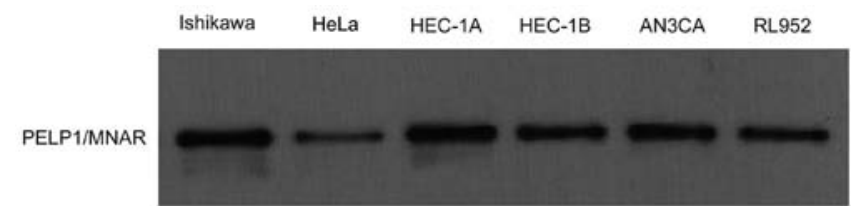

GAPDH
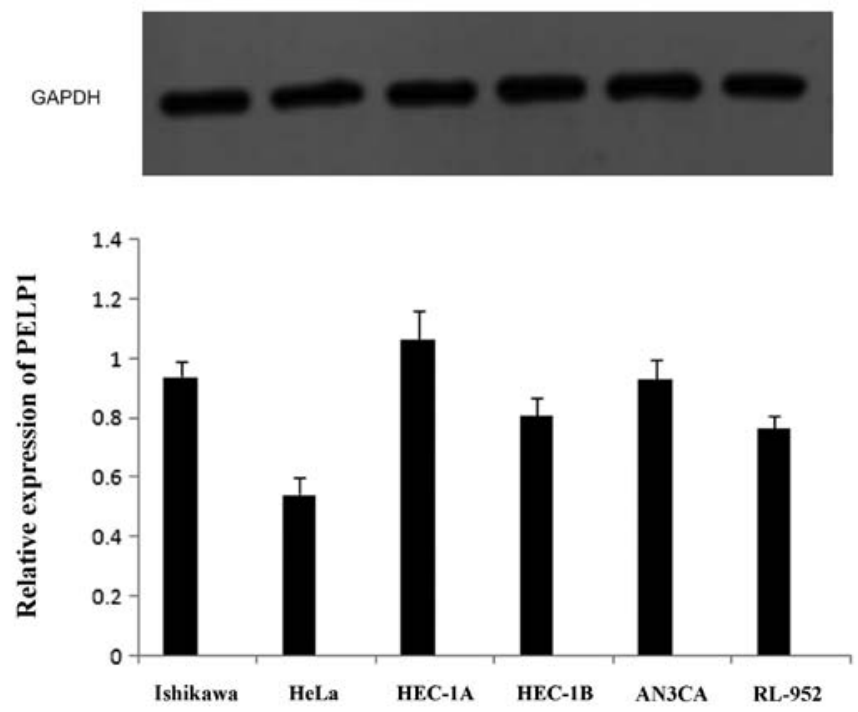

Figure 1. PELP1/MNAR expression in the Ishikawa, HEC-1A, HEC-1B, RL-952 and AN3CA endometrial cancer cell lines and the cervical cancer cell line, HeLa.

Downregulation of PELPI/MNAR $M R N A$ and protein expression in Ishikawa cells after lentivirus transfection.
To examine the function of endogenous PELP1/MNAR in endometrial cancer cells, we selectively knocked down the endogenous PELP1/MNAR expression using shRNA methodology. The fluorescent microphoto indicated that transfection was successful (Fig. 3). Additionally, flow cytometry analysis showed that the transfection efficiency was above $80 \%$. After 5 or 7 days of tranfection, the silencing effect was examined by real-time PCR and western blot analysis in the mock and stably transfected Ishikawa cells. As shown in Fig. 4, compared with the mock-transfected Ishikawa cells, the levels of PELP1/MNAR mRNA and protein in Ishikawa-KD cells were significantly reduced by 86 and $65 \%$, respectively $(\mathrm{P}<0.05)$; however, there was no significant difference between the Ishikawa-NC and Ishikawa-CON cells. These results confirmed the efficacy of lentivirus-mediated PELP1/MNARspecific shRNA in downregulating endogenous PELP1/MNAR expression.

PELP1/MNAR downregulation inhibits cell proliferation. To further analyze the effect of PELP1/MNAR downregulation on the proliferation of Ishikawa cells, we measured the proliferation rate of Ishikawa, Ishikawa-NC and Ishikawa-KD cells with or without $17 \beta-\mathrm{E} 2$ by MTT. The results showed that the proliferation of the Ishikawa-KD cells decreased in a timedependent manner compared with the parental cells and that the effect of PELP1/MNAR downregulation on cell proliferation was more pronounced when $17 \beta$-E2 was added (Fig. 5). We then analyzed the anchorage-dependent growth using a colony-forming assay. Similarly, Ishikawa-KD cells showed fewer colonies than Ishikawa-CON and Ishikawa-NC cells,
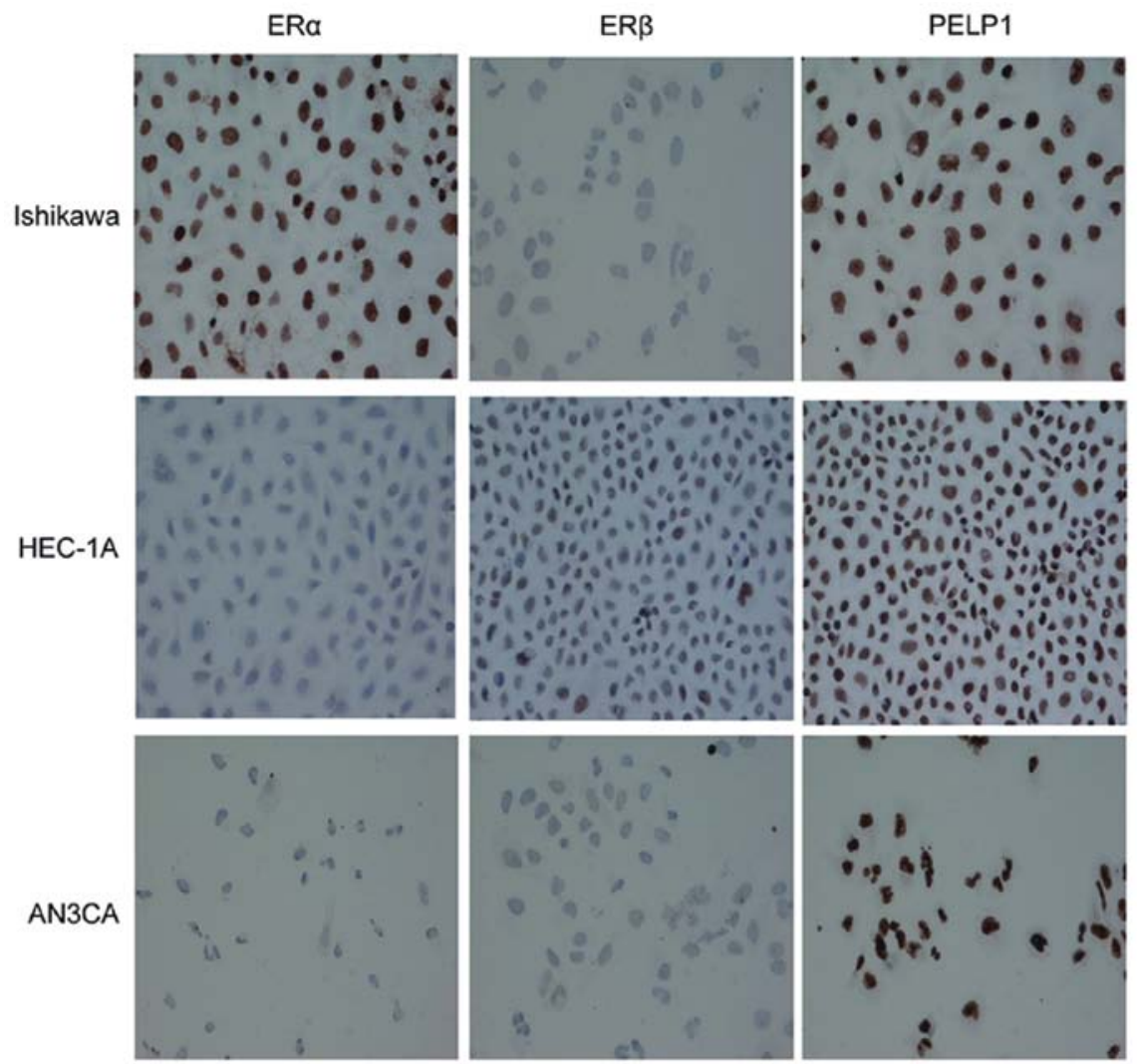

Figure 2. ER $\alpha, E R \beta$ and PELP1/MNAR expression in the Ishikawa, HEC-1A and AN3CA endometrial cancer cell lines. Magnification, $\mathrm{x} 400$. 

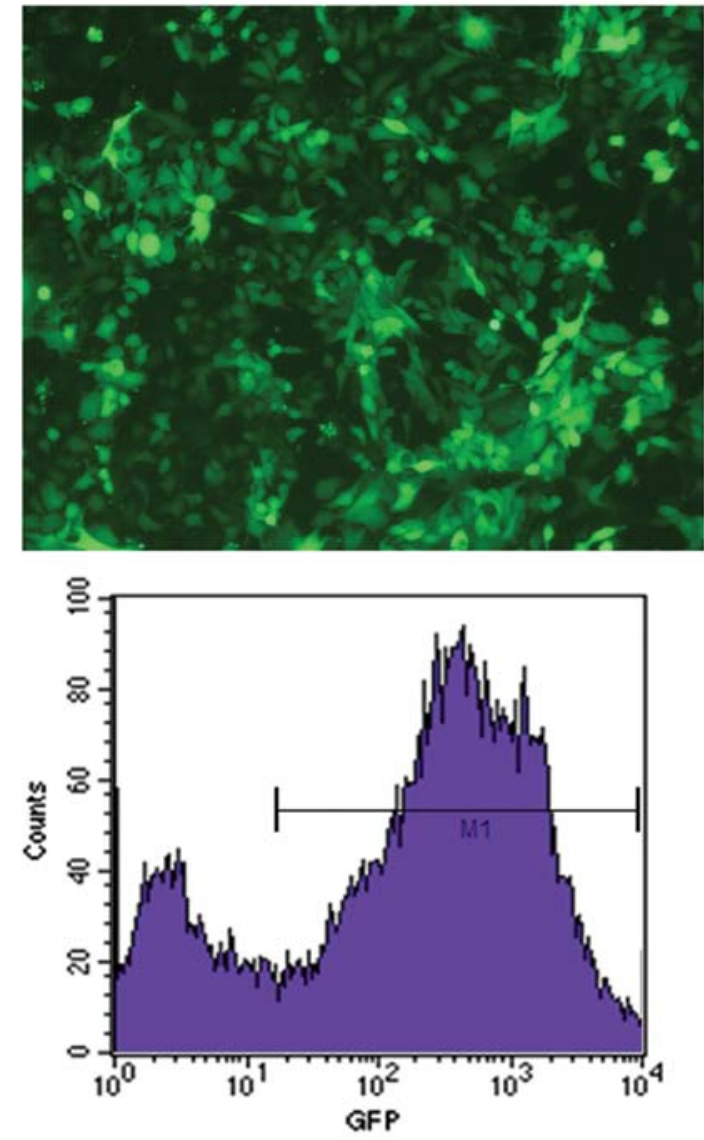

Ishikawa ( $50 \mathrm{MOI})$

\begin{tabular}{lllll}
\hline Marker & \% Gated & \% Total & Mean & Geo Mean \\
\hline All & 100 & 67.42 & 679.49 & 152.50 \\
M1 & 80.05 & 53.97 & 847.71 & 395.90
\end{tabular}

Figure 3. Fluorescent micrographs and flow cytometry analysis indicate transfection efficiency of Ishikawa cells transfected using a lentivirus vector expressing green fluorescent protein (GFP) after 96-h transfection. (Magnification, x100)

with or without E2 (10-9 M) treatment; however, there was no difference between the Ishikawa-NC and Ishikawa-CON cells (Fig. 6).

PELPI/MNAR downregulation reduces cell migration and invasion. To further examine whether the downregulation of PELP1/MNAR in Ishikawa cells reduces their migratory potential, we examined Ishikawa, Ishikawa-NC, Ishikawa-KD cells using Boyden chamber assay. Compared to the control cells, the knockdown of PELP1/MNAR in Ishikawa cells resulted in significantly reduced cell motility (Fig. 6). Moreover, the accelerated cell migratory effect of E2 was blocked in the Ishikawa-KD cells. The downregulation of PELP1/MNAR in the Ishikawa cells also significantly reduced the invasive potential, as shown by Boyden chamber assay (Fig. 7) and more importantly, abolished the E2-enhanced invasion $(\mathrm{P}<0.05)$. These results suggest that PELP1/MNAR plays an important role in cell invasion. To further understand the mechanism involved, we examined the expression of matrix metalloproteinase (MMP)-2 and MMP-9 to investigate
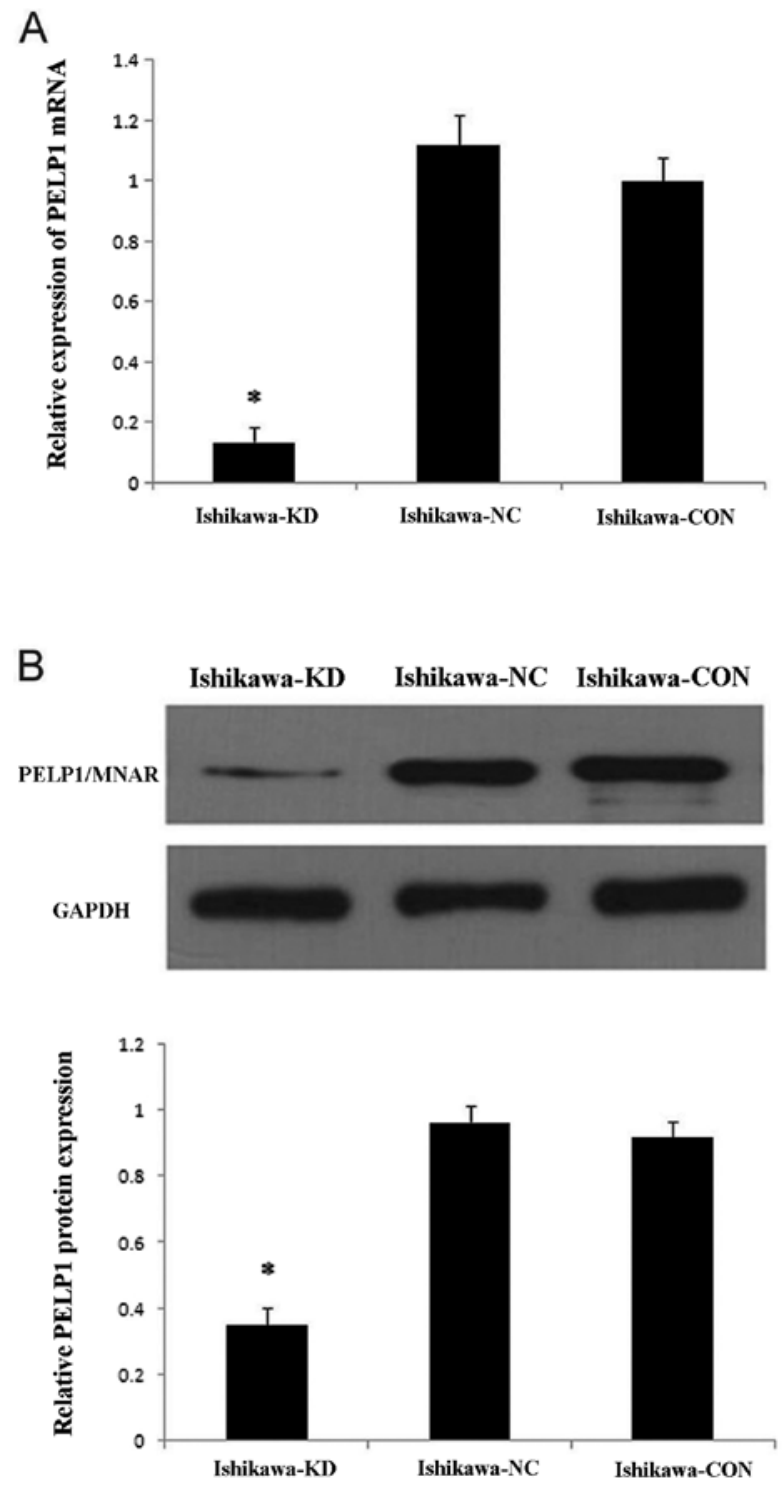

Figure 4. Detection of PELP1/MNAR mRNA and protein expression in Ishikawa cells after stable transfection with PELP1/MNAR-RNAi or negative control shRNA, respectively. (A) PELP1/MNAR and $\beta$-actin mRNA levels were determined by real-time PCR 5 days after transfection. Data were normalized to the $\beta$-actin signal. (B) Western blot analysis of PELP1/MNAR expression in Ishikawa cells 7 days after transfection. GAPDH was used as the internal control. All experiments were performed in triplicate. ${ }^{*} \mathrm{P}<0.05$.

whether PELP1/MNAR downregulation altered the expression of MMPs. MMPs promote cancer progression by enhancing the growth, migration, invasion and metastasis of endometrial cancer cells. Total RNA isolated from Ishikawa, Ishikawa-NC and Ishikawa-KD cells was used for real-time PCR and the results suggested that PELP1/MNAR downregulation substantially reduced the expression of MMP-2 and MMP-9 compared to their expression in the control cells (Fig. 8).

\section{Discussion}

Although estrogen and ER signaling is considered the classic etiological factor for endometrial tumorigenesis, the modulation mechanisms of estrogen that are involved in endometrial cancer remain unclear. NR co-regulators (co-activators and co-repressors) are essential elements in 

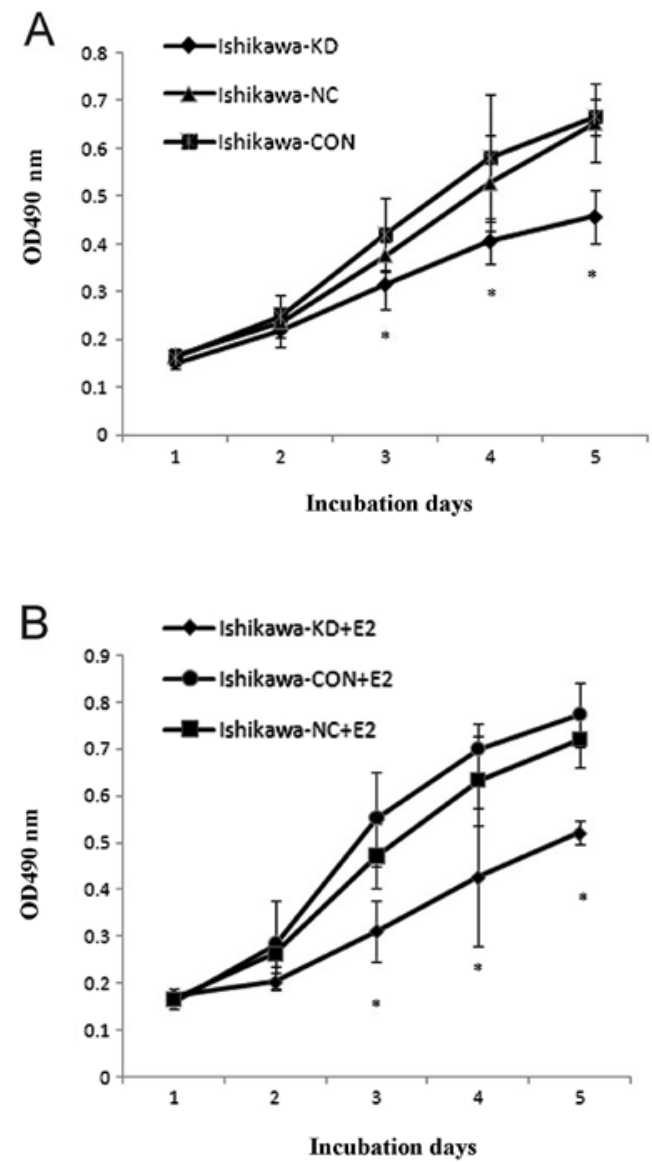

Figure 5. Effect of PELP1 knockdown on the proliferation of Ishikawa cells Seven days after transfection, Ishikawa cells were treated with or without E2 (10-9 M), and cell viability was measured using MTT assay on day 5 . The growth curves were applied to absorbance at $490 \mathrm{~nm}$. The results shown are 1 representative experiment from 3 experiments with similar results. Each point represents the means $\pm \mathrm{SD}$ of 3 independent experiments. ${ }^{*} \mathrm{P}<0.05$.

regulating NR-mediated transcription and other cellular events. Disruptions in co-regulator biology may lead to pathological states, including cancer. NR-co-regulator proteins have the potential to be differentially expressed in malignant tumors, and their functions may be altered, leading to tumor progression. The role of NR co-regulators as proto-oncogenes is an emerging area in the field of cancer research and, thus, represents a potential area for therapeutic targeting $(29,30)$. Several NR co-regulators reported to be misexpressed in endometrial cancer include amplified in breast cancer 1 (AIB1), metastasisassociated protein 1 (MTA1) and NR co-repressor ( $N c o R)$. The deregulation of PELP1/MNAR in endometrial carcinoma was also observed, which led us to hypothesize that PELP1/MNAR may play a role in endometrial carcinoma progression and that the downregulation of PELP1/MNAR may serve as a potential target for therapy.

To examine this hypothesis, we chose 5 endometrial cancer cell lines to investigate the expression of PELP1/MNAR, and found that PELP1/MNAR was widely expressed in endometrial cells, even in ER-negative cells, and the protein was mostly located in the nucleus. A recent study also showed that PELP1 expression was retained in ER-negative breast tumors. PELP1 knockdown reduced the motility and metastatic poten-
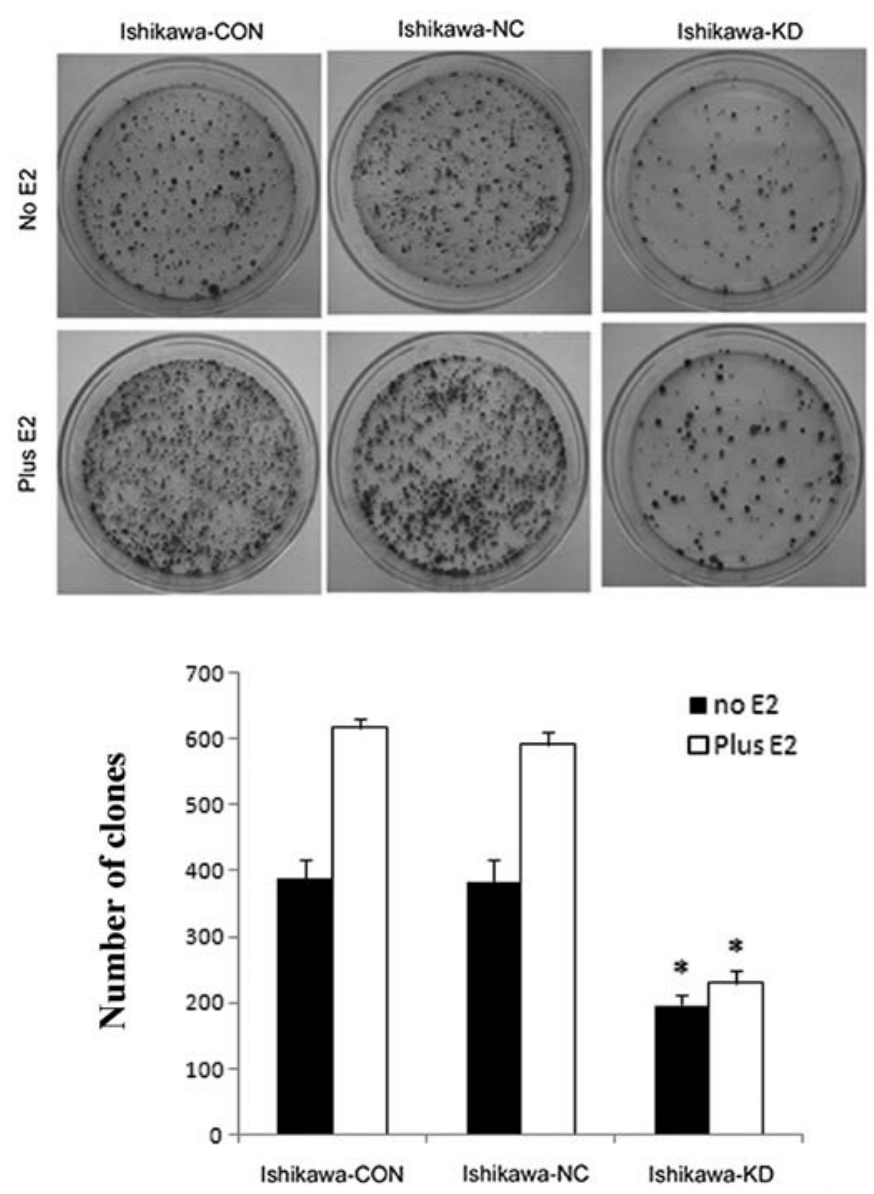

Figure 6. Effect of PELP1 knockdown on anchorage-dependent colony formation of Ishikawa cells. Five days after transfection, the 3 types of Ishikawa cells were plated in 6-cm dishes and treated with or without E2 (10-9 M), and cell colonies were counted after 14 days. Data are the means $( \pm$ SEM) of 3 different experiments in which each treatment was performed in triplicate within the experiment. ${ }^{*} \mathrm{P}<0.05$.

tial of ER-negative breast cancer cells in vivo and significantly reduced lung metastatic nodules in a xenograft assay. Their results suggested that PELP1 has potential to participate in hormone-driven pathologies and plays a role in the initiation and progression of ER-negative breast cancer (31). Whether or not PELP1 contributes to ER-negative endometrial carcinoma progression is yet to be determined; thus, further research is required. We then employed the newly developed lentivirus vector encoding shRNAs against PELP1/MNAR, which has high transfection efficiency and long duration resulting in stable ablation. After transfection, the endogenous expression of PELP1/MNAR was significantly knocked down in the commonly used endometrial carcinoma cell line, Ishikawa, in order to explore its role on the proliferation, invasion and metastasis of endometrial carcinoma cells.

Earlier studies have demonstrated that PELP1/MNAR is essential for the E2-mediated cell proliferation in several hormone-related cancers, including endometrial cancer. Mechanistic studies showed that PELP1/MNAR plays a permissive role in E2-mediated cell cycle progression by enhancing E2 mediated G1-S progression in breast cancer cells (32). A more recent study revealed that PELP1/MNAR is a novel substrate of interphase cyclin-dependent kinases (CDKs) and that its 

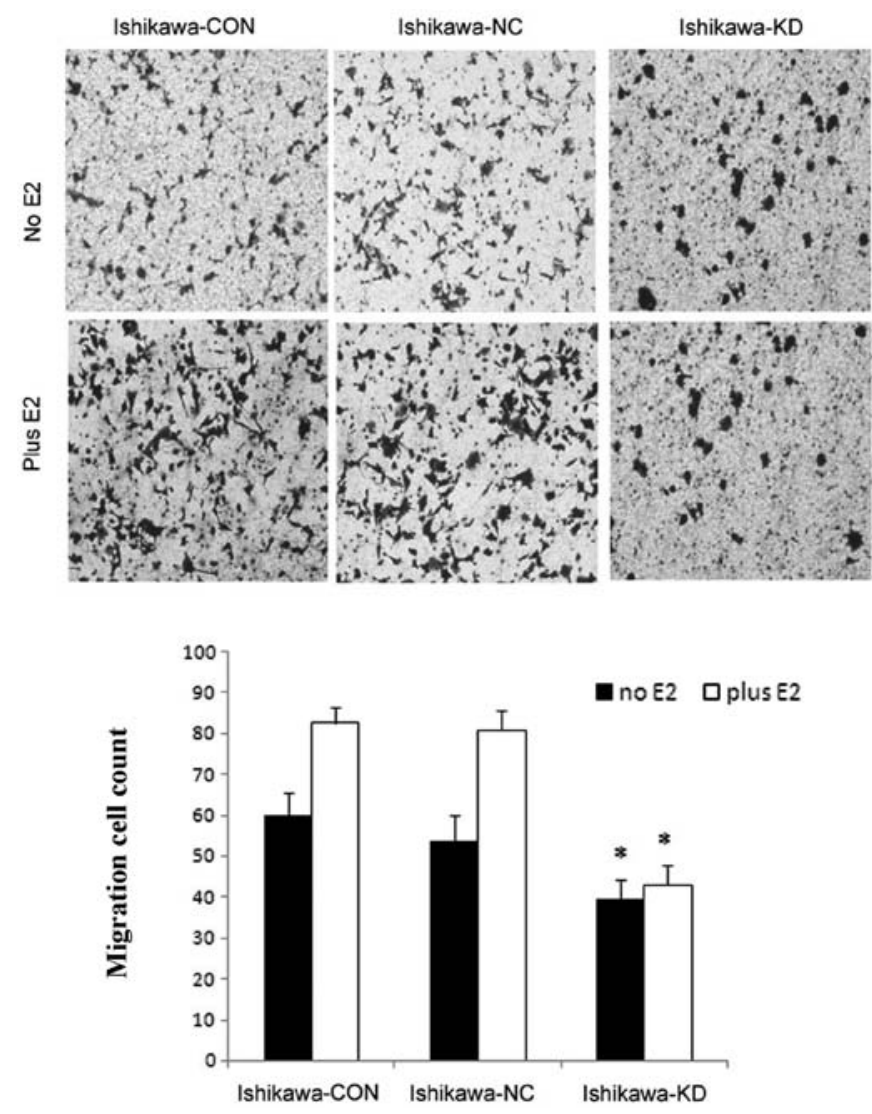

Figure 7. PELP1 knockdown affects the migration ability of Ishikawa cells Migratory potential of Ishikawa-CON, Ishikawa-NC and Ishikawa-KD cells were analyzed using Boyden chamber assay. Photomicrographs of migrated cells in various treatments are shown (magnification, $\mathrm{x} 100$ ). Data are the means $( \pm$ SEM) of 3 different experiments in which each treatment was performed in triplicate within the experiment. ${ }^{*} \mathrm{P}<0.05$.

phosphorylation is important for the proper function of PELP1/ MNAR in modulating hormone-driven cell cycle progression and also for optimal E2F transactivation function in breast cancer (33). In our study, the downregulation of PELP1/MNAR was accompanied by a significant suppression in the ability of estrogen to stimulate the growth of Ishikawa cells, as well as the basal proliferation without the addition of E2.

Evidence has shown that PELP1/MNAR also plays a role in cancer metastasis. PELP1/MNAR expression is deregulated in metastatic tumors $(26,31,34)$. PELP1/MNAR protein expression is an independent prognostic predictor of shorter breast cancer-specific survival, and its elevated expression is positively associated with markers of poor outcome (26). PELP1/MNAR plays a critical role in ovarian cancer cell migration and modulates the expression of several genes involved in metastasis (34), while its function in endometrial cancer remains unclear. In the present study, we found that the knockdown of PELP1/MNAR substantially affected the ability of endometrial cancer cells to migrate and invade, as shown by Boyden chamber assay. MMPs are a family of structurally related zinc- and calciumdependent endopeptidases that degrade extracellular matrix components. MMP-mediated proteolysis is involved in various physiological and pathological processes, such as cancer invasion and metastasis. MMP-2 and MMP-9 contribute to tumor invasion. Tumor invasion and angiogenesis are impaired by
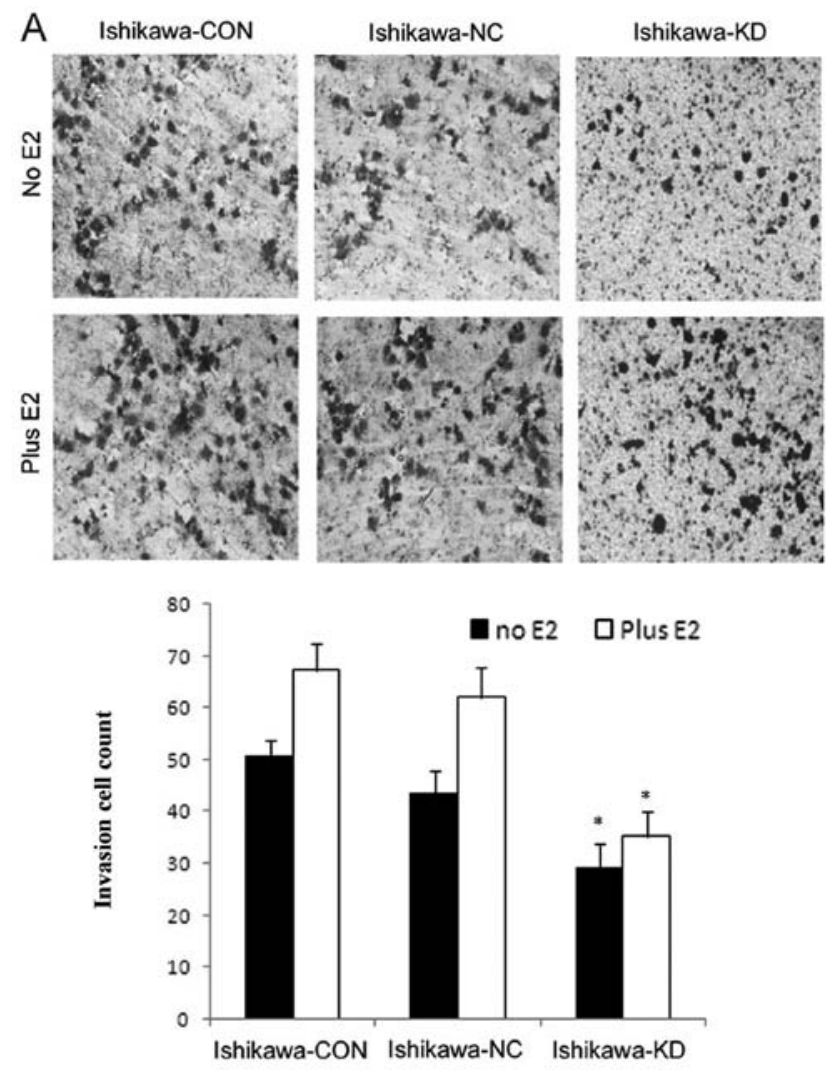

B

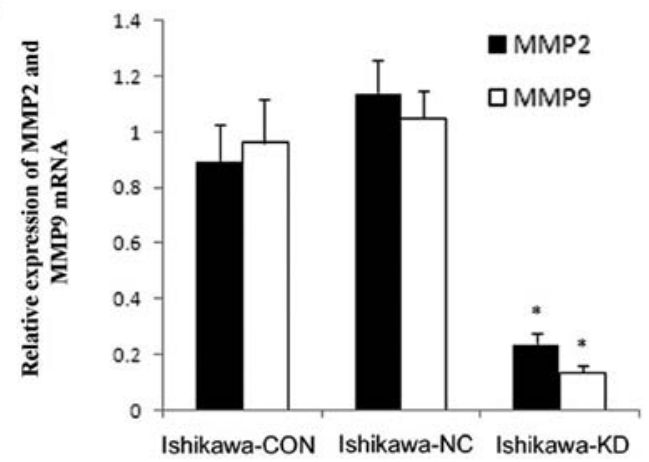

Figure 8. PELP1 knockdown affects the invasive potential of Ishikawa cells (A) Cell invasive potential of Ishikawa cells untransfected, transfected with control vector or PELP1 shRNA was analyzed using Matrigel invasion chamber assays. Photomicrographs of invaded cells in various treatments are shown (magnification, $x 100)$. Data are the means $( \pm$ SEM) of 3 different experiments in which each treatment was performed in triplicate within the experiment ${ }^{*} \mathrm{P}<0.05$. (B) Real-time PCR validation of the changes in MMP-2 and MMP-9 gene expression in Ishikawa-CON, Ishikawa-NC and Ishikawa-KD cells. Data are the means $( \pm$ SEM) of 3 different experiments in which each treatment was performed in triplicate within the experiment.

the combined deficiency in both metalloproteinases (35). Our results provide evidence that the production of MMP- 2 and MMP-9 was also decreased with PELP1/MNAR knockdown, which contributed to the inhibition of Ishikawa cell invasion.

Taken together, our study provides evidence that the downregulation of PELP1/MNAR inhibits the proliferation and metastasis of endometrial cancer cells in both an estrogendependent and estrogen-independent manner. The findings suggest that PELP1 may be used as a potential therapeutic target in endometrial cancer. Future study of the in vivo mechanisms of PELP1/MNAR activity and profile of the expression 
of PELP1/MNAR in a large number of tumor samples would confirm the use of this novel ER-co-regulatory protein as a diagnostic marker and as a target for novel therapies.

\section{Acknowledgements}

This study was supported by the Science and Technology Plan Project of Guang Dong Province (2007B030502014, 00429391120223052) and the National Nature Science Foundation of China (30772332).

\section{References}

1. Whitcomb BP: Gynecologic malignancies. Surg Clin North Am 88: 301-317,vi, 2008.

2. Zhang H: Endocrine-related cancer. In: Encyclopedia of Cancer. Schwab M (ed). 2nd edition. Springer-Verlag, Heidelberg, pp435-439, 2008.

3. Shang Y: Molecular mechanisms of oestrogen and SERMs in endometrial carcinogenesis. Nat Rev Cancer 6: 360-368, 2006.

4. Pietras RJ, Levin ER and Szego CM: Estrogen receptors and cell signaling. Science 310: 51-53, 2005.

5. Shao W and Brown M: Advances in estrogen receptor biology: prospects for improvements in targeted breast cancer therapy. Breast Cancer Res 6: 39-52, 2004.

6. O'Lone R, Frith MC, Karlsson EK and Hansen U: Genomic targets of nuclear estrogen receptors. Mol Endocrinol 18 $1859-1875,2004$

7. Bjornstrom L and Sjoberg M: Mechanisms of estrogen receptor signaling: convergence of genomic and non-genomic actions on target genes. Mol Endocrinol 19: 833-842, 2005.

8. Song RX, Zhang Z and Santen RJ: Estrogen rapid action via protein complex formation involving ERalpha and Src. Trends Endocrinol Metab 16: 347-353, 2005.

9. Losel $\mathrm{R}$ and Wehling $\mathrm{M}$ : Non-genomic actions of steroid hormones. Nat Rev Mol Cell Biol 4: 46-56, 2004.

10. Mckenna NJ, Lanz RB and O'Malley BW: Nuclear receptor coregulators: cellular and molecular biology. Endocr Rev 20: 321-344, 1999.

11. Hall JM and Mcdonnell DP: Coregulators in nuclear estrogen receptor action: from concept to therapeutic targeting. Mol Interv 5: 343-357, 2005.

12. Barnes CJ, Vadlamudi RK and Kumar R: Novel estrogen receptor coregulators and signaling molecules in human diseases. Cell Mol Life Sci 61: 281-291, 2004.

13. Han SJ, Demayo FJ, Xu J, Tsai SY, Tsai MJ and O'Malley BW: Steroid receptor coactivator (SRC)-1 and SRC-3 differentially modulate tissue-specific activation functions of the progesterone receptor. Mol Endocrinol 20: 45-55, 2006.

14. O'Malley BW: Coregulators: from whence came these 'master genes'. Mol Endocrinol 21: 1009-1013, 2007.

15. Sakaguchi H, Fujimto J and Sun WS: Clinical implications of steroid receptor coactivator (SRC)-3 in uterine endometrial cancers. J Steroid Biochem Mol Biol 104: 237-240, 2007.

16. Lanard DM, Lanz RB and O'Malhy BW: Nuclear receptor coregulators and human disease. Endocr Rev 28: 575-587, 2007.

17. Gururaj AE, Singh RR and Rayala SK: MTA1, a transcriptional activator of breast cancer amplified sequence 3. Proc Natl Acad Sci USA 103: 6670-6675, 2006.
18. Webb P, Valentine $\mathrm{C}$ and Nguyen P: ER beta binds N-CoR in the presence of estrogens via an LXXLL-like motif in the N-CoR C-terminus. Nucl Recept 1: 4, 2003.

19. Mishra SK, Balasenthil S and Ngnyen D: Cloning and functional characterization of PELP1/MNAR promoter. Gene 330: 115-122, 2004.

20. Nair SS, Nair BC, Cortez V, Chakravarty D, Metzger E, Schüle R, Brann DW, Tekmal RR and Vadlamudi RK: PELP1 is a reader of histone H3 methylation that facilitates oestrogen receptor-alpha target gene activation by regulating lysine demethylase 1 specificity. EMBO Rep 11: 438-444, 2010.

21. Cheskis B J, Greger J, Cooch N, et al: MNAR plays an important role in ER $\alpha$ activation of Src/MAPK and PI3K/Akt signaling pathways. Steroids 73: 901-905, 2008.

22. Rajhans R and Vadlamudi RK: Comprehensive analysis of recent biochemical and biologic findings regarding a newly discovered protein-PELP1/MNAR. Clin Exp Metastasis 23: 1-7, 2006.

23. Vadlamudi RK and Kumar R: Functional and biological properties of the nuclear receptor coregulator PELP1/MNAR. Nucl Recept Signal 5: e004, 2007.

24. Nair SS, Guo Z, Mueller J M, et al: PELP1/MNAR enhances androgen receptor functions through LIM-only coactivator FHL2. Mol Endocrinol 21: 613-624, 2007.

25. Rajhans R, Nair S, Holden AH, Kumar R, Tekmal RR and Vadlamudi RK: Oncogenic potential of the nuclear receptor coregulator proline-,glutamic acid-, leucine-rich protein 1/ modulator of the nongenomic actions of the estrogen receptor. Cancer Res 67: 5505-5512, 2007.

26. Habashy HO, Powe DG, Rakha EA, et al: The prognostic significance of PELP1 expression in invasive breast cancer with emphasis on the ER-positive luminal-like subtype. Breast Cancer Res Treat 120: 603-612, 2010.

27. Vadlamudi RK, Balasenthil S, Broaddus RR, Gustafsson JA and Kumar R: Deregulation of estrogen receptor coactivator proline-, glutamic acid-, and leucine-rich protein-1/modulator of nongenomic activity of estrogen receptor in human endometrial tumors. Clin Endocrinol Metab 89: 6130-6138, 2004.

28. Dimple C, Nair SS, Rajhans R, et al: Role of PELP1/MNAR signaling in ovarian tumorigenesis. Cancer Res 68: 4902-4909, 2008.

29. O'Malley BW and Kumar R: Nuclear receptor coregulators in cancer biology. Cancer Res 69: 8217-8222, 2009.

30. Chakravarty D, Tekmal RR and Vadlamudi RK: PELP1: a novel therapeutic target for hormonal cancers. IUBMB Life 62: 163-169, 2010.

31. Roy S, Chakravarty D, Cortez V, De Mukhopadhyay K, Bandyopadhyay A, Ahn JM, Raj GV, Tekmal RR, Sun L and Vadlamudi RK: Significance of PELP1 in ER-negative breast cancer metastasis. Mol Cancer Res 10: 25-33, 2012.

32. Balasenthil S and Vadlamudi RK: Functional interactions between the estrogen receptor coactivator PELP1/MNAR and retinoblastoma protein. J Biol Chem 278: 22119-22127, 2003.

33. Nair BC, Nair SS, Chakravarty D, et al: Cyclin-dependent kinase-mediated phosphorylation plays a critical role in the oncogenic functions of PELP1. Cancer Res 70: 7166-7175, 2010.

34. Chakravarty D, Roy SS, Babu CR, Dandamudi R, Curiel TJ, Vivas-Mejia P, Lopez-Berestein G, Sood AK and Vadlamudi RK: Therapeutic targeting of PELP1 prevents ovarian cancer growth and metastasis. Clin Cancer Res 17: 2250-2259, 2011.

35. Egeblad M and Werb Z: New functions for the matrix metalloproteinases in cancer progression. Nat Rev Cancer 2: 161-174, 2002. 